\title{
Boosting Tissue Vascularization: Nanofat as a Potential Source of Functional Microvessel Segments
}

\author{
Andrea Weinzierl ${ }^{1 *}$, Yves Harder ${ }^{2,3}$, Daniel Schmauss ${ }^{2,3}$, Michael D. Menger ${ }^{1}$ and \\ Matthias W. Laschke ${ }^{1}$
}

${ }^{1}$ Institute for Clinical \& Experimental Surgery, Saarland University, Homburg, Germany, ${ }^{2}$ Department of Plastic, Reconstructive and Aesthetic Surgery, Ospedale Regionale di Lugano, Ente Ospedaliero Cantonale (EOC), Lugano, Switzerland, ${ }^{3}$ Faculty of Biomedical Sciences, Università Della Svizzera Italiana, Lugano, Switzerland

\section{OPEN ACCESS}

Edited by:

Arnaud Scherberich,

University Hospital of Basel,

Switzerland

Reviewed by:

Ziqing Dong,

Southern Medical University, China

Timo Ten Hagen,

Erasmus University Medical Center,

Netherlands

*Correspondence:

Andrea Weinzier

andreaweinzier/@icloud.com

Specialty section:

This article was submitted to

Tissue Engineering and Regenerative

Medicine,

a section of the journal Frontiers in Bioengineering and

Biotechnology

Received: 23 November 2021

Accepted: 14 January 2022

Published: 04 February 2022

Citation:

Weinzierl A, Harder Y, Schmauss D,

Menger MD and Laschke MW (2022)

Boosting Tissue Vascularization:

Nanofat as a Potential Source of

Functional Microvessel Segments.

Front. Bioeng. Biotechnol. 10:820835.

doi: 10.3389/fbioe.2022.820835
Nanofat is increasingly applied in plastic surgery for the improvement of scar quality and skin rejuvenation. However, little is known about the underlying regenerative mechanisms. Therefore, we herein investigated nanofat grafts in a murine dorsal skinfold chamber model. Nanofat generated from subcutaneous, inguinal adipose tissue of green fluorescent protein (GFP) ${ }^{+}$C57BL/6 male and female donor mice was injected intracutaneously into dorsal skinfold chambers of gender-matched GFP ${ }^{-}$wild-type mice. The vascularization and tissue composition of the grafted nanofat were analyzed by means of intravital fluorescence microscopy, histology and immunohistochemistry over an observation period of 14 days. The freshly generated nanofat consisted of small fragments of perilipin ${ }^{+}$adipocytes surrounded by Sirius red ${ }^{+}$collagen fibers and still contained intact $\mathrm{CD} 31^{+} / \mathrm{GFP}^{+}$vessel segments. After transplantation into the dorsal skinfold chamber, these vessel segments survived and developed interconnections to the surrounding $\mathrm{CD}_{3} 1^{+} / \mathrm{GFP}^{-}$host microvasculature. Accordingly, the grafted nanofat rapidly vascularized and formed new microvascular networks with a high functional microvessel density on day 14 without marked differences between male and female mice. Even though further research is needed to confirm these findings, the present study suggests that nanofat boosts tissue vascularization. Thus, nanofat may represent a versatile resource for many applications in tissue engineering and regenerative medicine.

Keywords: fat graft, nanofat, vascularization, dorsal skinfold chamber, intravital fluorescence microscopy, tissue engineering

\section{INTRODUCTION}

The first recorded fat graft was executed in 1893 by the German surgeon Gustav Neuber to correct adherent osteomyelitis-induced scar sequelae (Mazzola and Mazzola, 2015). After decades of using mainly en bloc fat grafts for the correction of contour deformities, the introduction of liposuction (Illouz, 1986) in the 1980s finally paved the way for modern fat grafting by means of easily reinjectable, aspirated microfat. Since then, microfat grafts have also been increasingly used due to their beneficial effects on damaged tissue, such as burn scars (Mazzola and Mazzola, 2015). The true regenerative potential of fat grafts was ultimately confirmed in the early 2000s, when the presence of mesenchymal stem cells was discovered in adult adipose tissue (Zuk et al., 2001; Zuk et al., 2002). Nowadays, microfat grafts are frequently 
used for breast reconstruction. However, this procedure bears the risk of graft absorption due to an insufficient vascularization.

In 2013, Tonnard et al. (2013) introduced the technique of nanofat grafting. This processing method uses emulsification and filtering of microfat to burst the majority of mature adipocytes, resulting in grafts that mostly consist of released growth factors and adipose-derived stem cells (ASCs). Hence, the principal aim of nanofat grafting is not the restoration of volume, but the induction of tissue remodeling and the improvement of tissue quality. In this context, it should be considered that adipose tissue does not only contain adipocytes and ASCs, but also various other cell types, such as fibroblasts and immune cells, as well as extracellular matrix and microvessels (Brüggen and Stingl, 2020; Chait and den Hartigh, 2020; Herold and Kalucka, 2021). However, the effects of these other components on the engraftment of nanofat remain largely unknown. Since vascularization is a major determinant for adequate tissue regeneration (Banfi et al., 2018; Laschke and Vollmar, 2018; Gianni-Barrera et al., 2020), it may be particularly interesting to study the fate of microvessels during the process of nanofat generation and transplantation. Given that the fabrication of nanofat only applies mechanical forces to break down the tissue, it is unlikely that individual cell-cell bonds are broken, as it is the case for the enzymatic digestion of adipose tissue into single stromal vascular fraction (SVF) cells (Zuk et al., 2001). Therefore, we herein hypothesized that nanofat still contains functional vessel segments that are able to rapidly reassemble into new microvascular networks.

It is well known that estrogen is a major promotor for angiogenesis and tissue vascularization (Rubanyi et al., 2002). In the context of fat grafting, this is demonstrated by the fact that fat grafts from non-ovariectomized mice are more vascularized when compared to ovariectomized animals (Bills et al., 2015). Furthermore, Mok et al. (2018) reported that elevated serum estrogen increases ASC density and early vascularization in microfat grafts. Similar results were observed by $\mathrm{Wu}$ et al. (2020), who detected a significantly higher expression of estrogen receptor $a$ and a concomitantly elevated vascular density in grafted adipose tissue from animals receiving estradiol treatment. Based on these findings, we further speculated that the vascularization of grafted nanofat is improved in females.

To test our hypotheses in the present study, we generated nanofat from subcutaneous, inguinal adipose tissue of green fluorescent protein $(\mathrm{GFP})^{+} \mathrm{C} 57 \mathrm{BL} / 6$ male and female donor mice. The nanofat was subsequently injected intracutaneously into dorsal skinfold chambers of gender-matched $\mathrm{GFP}^{-}$wildtype animals to assess its vascularization and tissue composition by means of intravital fluorescence microscopy, histology and immunohistochemistry over an observation period of 14 days.

\section{MATERIALS AND METHODS}

\section{Animals}

All animal experiments were approved by the local governmental animal protection committee (permit number: 30/2020) and conducted in accordance with the European legislation on the protection of animals (Directive 2010/63/EU) and the NIH Guidelines on the Care and Use of Laboratory Animals (NIH publication \#85-23 Rev. 1985).

In this study, 4 male and 4 female $\mathrm{GFP}^{+}$mice $(\mathrm{C} 57 \mathrm{BL} / 6-\mathrm{Tg}$ (CAG-EGFP)1Osb/J; The Jackson Laboratory, Bar Harbor, ME, United States) with an age of up to 52 weeks and a body weight of 28-32 g served as gender-matched fat donors for the generation of nanofat in sufficient amounts. The nanofat was grafted into the dorsal skinfold chambers of 8 male and 8 female GFP ${ }^{-}$C57BL/ 6 wild-type mice (Institute for Clinical \& Experimental Surgery, Saarland University, Homburg/Saar, Germany) with an age of $12-20$ weeks and a body weight of $23-26 \mathrm{~g}$. The $\mathrm{GFP}^{+} / \mathrm{GFP}^{-}$ cross-over design of the present study allowed the differentiation of $\mathrm{GFP}^{+}$cells originating from the nanofat grafts and $\mathrm{GFP}^{-}$cells from the host tissue within the dorsal skinfold chamber.

The animals were kept at a room temperature of $22-24^{\circ} \mathrm{C}$ and a 12-h day-night cycle. They had free access to standard pellet chow (Altromin, Lage, Germany) and tap water. Mice with a dorsal skinfold chamber were kept one per cage for the duration of the experiments.

\section{Anesthesia}

Harvesting of adipose tissue, dorsal skinfold chamber implantation and intravital fluorescence microscopy were performed in general anesthesia induced by intraperitoneal injection of ketamine $\left(100 \mathrm{mg} / \mathrm{kg}\right.$ body weight; Ursotamin ${ }^{\circledR}$; Serumwerke Bernburg, Bernburg, Germany) and xylazine $\left(12 \mathrm{mg} / \mathrm{kg}\right.$ body weight; Rompun ${ }^{\circledR}$; Bayer, Leverkusen, Germany). All animals received a subcutaneous injection of $5 \mathrm{mg} / \mathrm{kg}$ carprofen (Rimadyl ; Zoetis Deutschland $\mathrm{GmbH}$, Berlin, Germany) during the chamber implantation to prevent postoperative pain.

\section{Generation of Nanofat}

The inguinal subcutaneous adipose tissue (Figure 1A) of anesthetized $\mathrm{GFP}^{+}$donor mice was excised, taking care not to include the inguinal lymph node. The tissue was then minced by means of a histology tissue cutter (McIlwain Tissue Chopper, CLE Co. Ltd., Gomshall, UK) to produce fat fragments of an identical volume $(1 \times 1 \times 1 \mathrm{~mm})$, which were rinsed in $0.9 \% \mathrm{NaCl}$ solution. For emulsification, the fat was shuffled between two syringes using three female-to-female Luer lock connectors with descending internal diameters of $2.4,1.4$ and $1.2 \mathrm{~mm}$ and 30 passes per connector (Figure 1B). In a last step, the fat was passed through a cell filter with $500 \mu \mathrm{m}$ pore size to remove any larger remaining fat particles or debris (Figure 1C).

\section{Dorsal Skinfold Chamber Model}

A dorsal skinfold chamber was implanted into $\mathrm{GFP}^{-}$recipient mice, as described previously in detail (Laschke et al., 2011). For this purpose, the extended skinfold was sandwiched between two titanium chamber frames (Irola Industriekomponenten $\mathrm{GmbH} \&$ Co. KG, Schonach, Germany). One layer of skin was completely removed in a circular area of $\sim 15 \mathrm{~mm}$ in diameter. The remaining layers of striated panniculus carnosus muscle, subcutaneous tissue and skin served as the host tissue for the nanofat grafts and were sealed with a removable cover glass, which was fixed by 


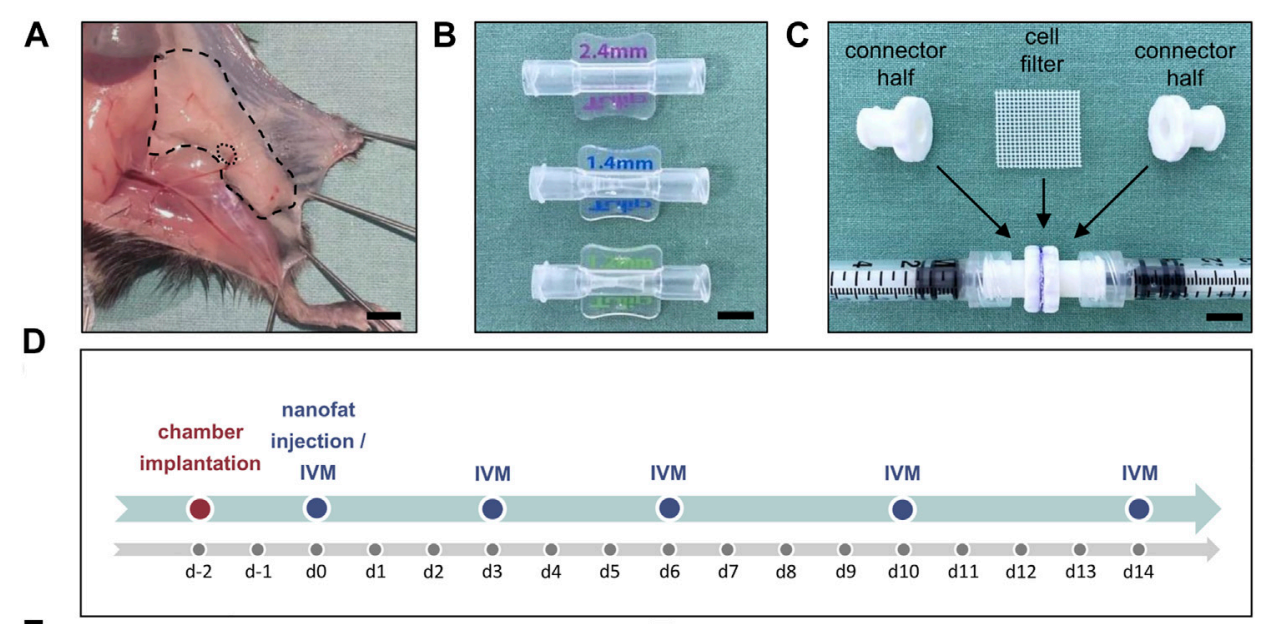

E
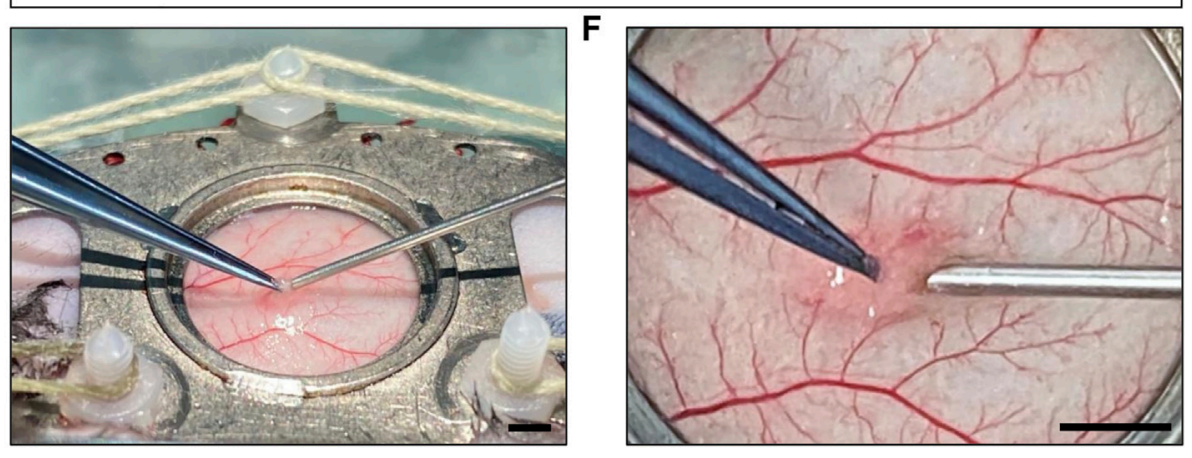

FIGURE 1 | (A) Inguinal subcutaneous adipose tissue (borders marked by broken line) with its inguinal lymph node (borders marked by dotted line) for the generation of nanofat. Scale bar: $5 \mathrm{~mm}$. (B) Luer lock connectors with descending internal diameters of $2.4 \mathrm{~mm}$ (top), $1.4 \mathrm{~mm}$ (middle) and 1.2 mm (below) used for fat emulsification. Scale bar: 5 mm. (C) Cell filter (500 $\mu \mathrm{m}$ pore size) sandwiched between two Luer lock connector halves to filter the emulsified fat. Scale bar: 5 mm. (D) Experimental protocol of the present study. The implantation of the dorsal skinfold chamber was performed $48 \mathrm{~h}$ prior to nanofat injection in male $(n=8)$ and female $(n=8)$ mice. Graft vascularization was repeatedly analyzed by means of intravital fluorescence microscopy (IVM) on day $0,3,6,10$ and 14 after transplantation. (E) Overview of the dorsal skinfold chamber during nanofat injection with a $27 \mathrm{G}$ needle. Scale bar: 2 mm. (F) Higher magnification of the graft injection site within the observation window. Scale bar: $2 \mathrm{~mm}$.

a snap ring. Following this implantation procedure, the animals were allowed to recover for $48 \mathrm{~h}$ to exclude alterations of the microcirculation due to anesthesia or surgical trauma (Figure 1D). Subsequently, the recipient mice were anesthetized again, the cover glass of chamber window was removed and $10 \mu \mathrm{L}$ of freshly prepared nanofat were injected into the subcutis underneath the panniculus carnosus muscle using a $27 \mathrm{G}$ needle (Figures 1E,F). Thereafter, the chamber was closed with a new cover glass and the snap ring. The remainder of the prepared nanofat was embedded in $1 \%$ agarose for histological and immunohistochemical analyses. All animals tolerated the chamber implantation and nanofat grafts well and showed normal feeding and sleeping habits during the observation period.

\section{Intravital Fluorescence Microscopy}

Repeated intravital fluorescence microcopy was performed directly after nanofat grafting (day 0 ) as well as on days 3, 6, 10 and 14 (Figure 1D). The microscopy was conducted before and after the retrobulbar injection of $0.1 \mathrm{ml}$ of the blood plasma marker 5\% fluorescein isothiocyanate (FITC)-labeled dextran
(150,000 Da; Sigma-Aldrich, Taufkirchen, Germany) for contrast enhancement. For this purpose, the anesthetized animals were fixed on a plexiglass stage and placed under a Zeiss Axiotech fluorescence epi-illumination microscope (Zeiss, Oberkochen, Germany). The nanofat grafts were recorded with a charge-coupled device video camera (FK6990; Pieper, Schwerte, Germany) and a DVD system. By means of $5 x, \times 10$ and $\times 20$ longdistance objectives (Zeiss) magnifications of $\times 115, \times 230$ and $\times 460$ were achieved on a 14 -inch video screen (Trinitron, Sony, Tokyo, Japan).

The microscopic images were analyzed by means of the offline analysis system CapImage (Version 8.5, Zeintl, Heidelberg, Germany). The analysis included the quantitative assessment of the perfused graft surface (in \% of the total graft surface). Moreover, the functional microvessel density (FMD), i.e. the overall length of all red blood cell (RBC)-perfused microvessels per observation area (in $\mathrm{cm} / \mathrm{cm}^{2}$ ), was measured in 5 regions of interest (ROI; $\times 460$ magnification) within the grafts. Furthermore, the fraction of ROI with $\mathrm{GFP}^{+}$bloodperfused microvessels out of all ROI (in \%) was assessed. In addition, microhemodynamic parameters were measured in up to 
5 randomly chosen microvessels per ROI, as soon as blood perfusion could be detected. Vessel diameters $(D$, in $\mu \mathrm{m})$ were measured perpendicular to the vessel path. The centerline RBC velocity (V, in $\mu \mathrm{m} / \mathrm{s}$ ) was assessed using the line shift method (De Vriese et al., 2000). The volumetric blood flow (VQ, in $\mathrm{pL} / \mathrm{s}$ ) was calculated from $\mathrm{V}$ and $\mathrm{D}$ as $\mathrm{VQ}=\pi \times(\mathrm{D} / 2) 2 \times \mathrm{V} / \mathrm{K}$ where $\mathrm{K}=1.6$ represents the Baker-Wayland factor considering the parabolic velocity profile of blood in microvessels (Baker and Wayland, 1974). Additionally, the measured microhemodynamic parameters were used to calculate the wall shear rate ( $y$, in $\mathrm{s}^{-1}$ ) by means of the Newtonian definition $\mathrm{y}=8 \times \mathrm{V} / \mathrm{D}$.

After the last microscopy, the animals were euthanized by cervical dislocation and the dorsal skinfold chamber preparations were carefully excised for further histological and immunohistochemical analyses.

\section{Histology and Immunohistochemistry}

Samples of freshly generated nanofat as well as dorsal skinfold chamber preparations with nanofat grafts were fixed in paraformaldehyde, embedded in paraffin and cut into 3- $\mu \mathrm{m}$ thick sections. Hematoxylin and eosin (HE) as well as Sirius red stainings of individual sections were performed according to standard procedures.

For the immunohistochemical detection of adipocytes, tissue sections were stained with a monoclonal rabbit anti-mouse antibody against perilipin (1:200; Cell Signaling Technology, Danvers, United States) as primary antibody followed by a goat anti-rabbit peroxidase-labeled antibody (1:100; Jackson ImmunoResearch Laboratories, West Grove, United States). The used chromogen was 3-amino-9-ethylcarbazole (Abcam, Cambridge, United Kingdom). All sections were counterstained with Mayer's hemalum (Merck, Darmstadt, Germany).

For the immunohistochemical detection of endothelial cells, tissue sections were stained with a monoclonal rat anti-mouse antibody against CD31 (1:100; Dianova, Hamburg, Germany) as primary antibody and a goat anti-rat Alexa 555 antibody (1:100; Invitrogen, Waltham, United States) as secondary antibody. To determine the origin of the endothelial cells, the sections were additionally stained with a polyclonal GFP goat antibody (1:100; Rockland Immunochemicals Inc., Limerick, United States) followed by a donkey-anti-goat biotin-labeled antibody (1:100; Life Technologies, Carlsbad, United States) and Alexa 488labeled streptavidin (1:50; Invitrogen). On each section, cell nuclei were stained with Hoechst 33,342 $(2 \mu \mathrm{L} / \mathrm{ml}$; SigmaAldrich) to merge images exactly.

All sections were assessed using a BX60 microscope (Olympus, Hamburg, Germany) and the imaging software cellSens Dimension 1.11 (Olympus). The number of $\mathrm{CD}^{+} 1^{+}$vessel segments was quantified in 3 high-power fields (HPF) per tissue specimen of freshly generated nanofat using the image processing software Fiji (open source software). In addition, we measured the fraction of $\mathrm{CD} 1^{+} / \mathrm{GFP}^{+}$microvessels out of all $\mathrm{CD} 31^{+}$microvessels in $3 \mathrm{HPF}$ per nanofat graft in the dorsal skinfold chamber.

\section{Statistical Analysis}

After testing the data for normal distribution and equal variance, differences between the two groups were analyzed by the unpaired Student's t-test (GraphPad Prism 9; GraphPad Software, San Diego, United States). In case of non-parametric data, a Mann-Whitney rank sum test was used. All values are expressed as means \pm standard error of the mean (SEM). Statistical significance was accepted for a value of $p<0.05$.

\section{RESULTS}

\section{Characterization of Freshly Generated Nanofat}

The cellular composition and morphology of freshly generated nanofat was analyzed by means of histology and immunohistochemistry. The effects of emulsification were clearly visible by the presence of ruptured adipocytes and cellular debris throughout the grafts (Figure 2A). Of interest, the nanofat also contained significant amounts of Sirius $\mathrm{red}^{+}$ collagen fibers of the extracellular matrix and intact perilipin ${ }^{+}$ adipocytes (Figure 2A). Between these adipocytes, well preserved $\mathrm{CD} 31^{+}$vessel segments could be detected (Figure 2B). In freshly generated nanofat from both male and female donor mice $\sim 60$ vessel segments per HPF could be counted (Figure 2C).

\section{In Vivo Vascularization of Grafted Nanofat}

The nanofat grafts were analyzed by means of repeated stereomicroscopy and intravital fluorescence microscopy within the dorsal skinfold chambers of male and female recipient mice. All grafts survived the transplantation procedure and could be easily detected within the chamber window throughout the observation period of 14 days (Figure 2D).

Graft vascularization did not markedly differ between male and female mice. Directly after transplantation, the grafts of both groups exhibited a completely disintegrated tissue architecture with cells, cellular debris and oil or fluid vacuoles of varying sizes without any distinct organization (Figure 3A). First bloodperfused microvessels could already be detected on day 3 . Throughout the following days, a progressively increasing number of blood-perfused microvessels was found within the grafts. These microvessels finally formed microvascular networks with an FMD of $\sim 280 \mathrm{~cm} / \mathrm{cm}^{2}$ on day 14 (Figures 3B,C). Between these networks isolated adipocytes, small adipocyte clusters and remnants of not yet absorbed oil vacuoles could still be identified (Figure 3A). The vascularization process was slightly accelerated in female animals when compared to male mice, as indicated by a higher perfused graft area and FMD on day 6, which, however, did not prove to be significant (Figures 3B,C). The additional assessment of microhemodynamic parameters revealed that the diameter and VQ of blood-perfused microvessels remained relatively constant over time (Figures 3D,F), whereas the centerline RBC velocity and shear rate slightly increased until the end of the experiments (Figures 3E,G).

In both groups, blood-perfused $\mathrm{GFP}^{+}$microvessels could be observed in the grafted nanofat. These $\mathrm{GFP}^{+}$microvessels could be further subdivided into arterioles, capillaries and venules by their distinct morphology and blood flow direction (Figure 4A). Of interest, at later observation time points the $\mathrm{GFP}^{+}$microvessels 
A
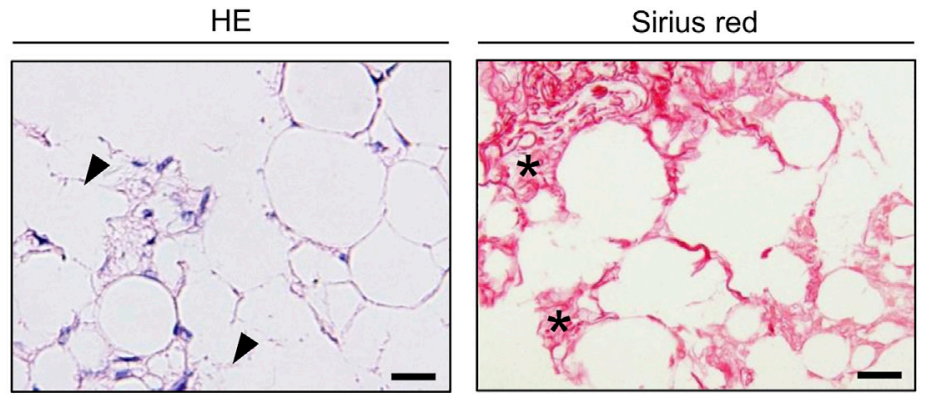

B

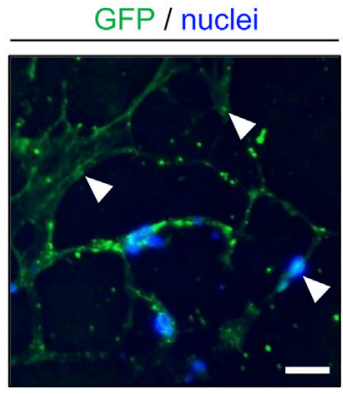

dO

D
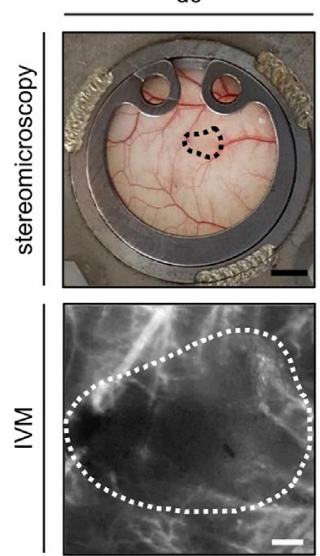

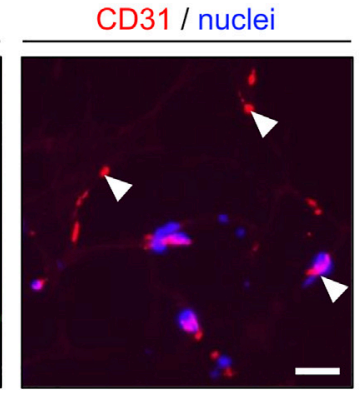

d3
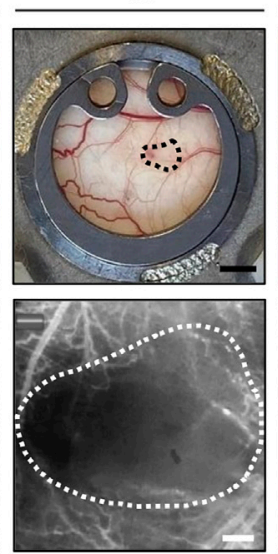
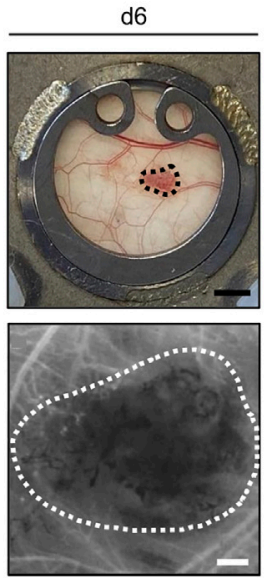

merged

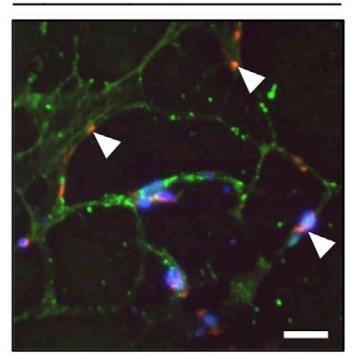

C

d10
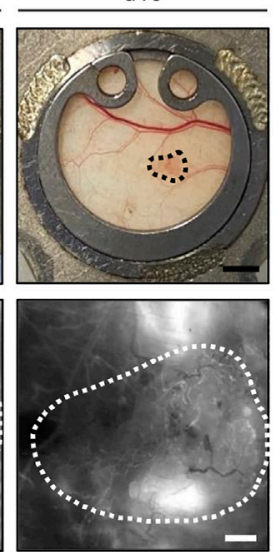

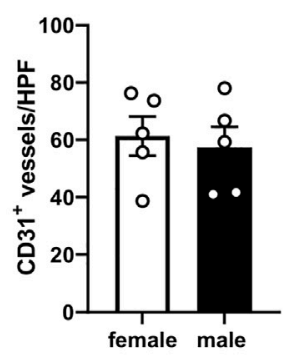

perilipin
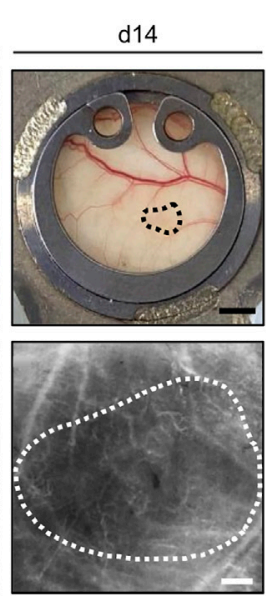

FIGURE 2 | (A) Histological and immunohistochemical stainings of freshly isolated nanofat exhibiting remnants of burst adipocytes and cell detritus (HE; arrowheads), Sirius red ${ }^{+}$collagen fibers (asterisks) and intact perilipin ${ }^{+}$adipocytes (hashtags). Scale bars: 25 um. (B) Immunofluorescent CD31/GFP stainings of freshly isolated nanofat containing intact vessel segments (arrowheads) in between and around adipocytes and adipocyte remnants. Cell nuclei are stained with Hoechst 33,342 (blue). Scale bars: $25 \mu \mathrm{m}$. (C) Number of CD31+ vessels per HPF in freshly generated nanofat from female ( $n=5$; white bars) and male $(n=5$; black bars) donor mice. (D) Stereomicroscopic and intravital fluorescence microscopic (IVM) images of a nanofat graft (borders marked by dotted line) within the dorsal skinfold chamber of a female mouse on day 0, 3, 6, 10 and 14 after transplantation. Scale bars (upper panels): 2 mm; scale bars (lower panels): 200 um. Means \pm SEM.

grew out of the grafts into the surrounding host tissue (Figure 4B), which demonstrates their tissue vascularization capacity. The presence of $\mathrm{GFP}^{+}$microvessels was detected as early as day 6 and in up to $70 \%$ of the analyzed ROI by day 14 (Figure 4C).

\section{Incorporation of Grafted Nanofat}

At the end of the in vivo experiments, additional histological and immunohistochemical analyses were performed to examine the morphology and incorporation of the nanofat grafts. HE-stained tissue sections confirmed that the grafts were localized in the subcutis between the dermis and panniculus carnosus muscle (Figure 5A). On day 14, they still consisted of microvessels, fibrous tissue and perilipin ${ }^{+}$ adipocytes (Figures 5A-C).

More detailed immunohistochemical analyses for the determination of vessel origin demonstrated that the nanofat grafts contained $\mathrm{CD} 1^{+} / \mathrm{GFP}^{+}$microvessels, which developed interconnections to the $\mathrm{CD}^{+} 1^{+} / \mathrm{GFP}^{-}$microvessels of the surrounding tissue (Figures 6A,B). In line with our intravital fluorescent microscopic findings, we further detected $\mathrm{CD}_{3} 1^{+} /$ $\mathrm{GFP}^{+}$microvessels outside the grafts, resulting in an improved vascularization of the transplantation site (Figure 6C). These $\mathrm{CD} 1^{+} / \mathrm{GFP}^{+}$microvessels made up $\sim 40 \%$ of all $\mathrm{CD} 31^{+}$ microvessels without statistical differences between male and female animals (Figure 6D). 

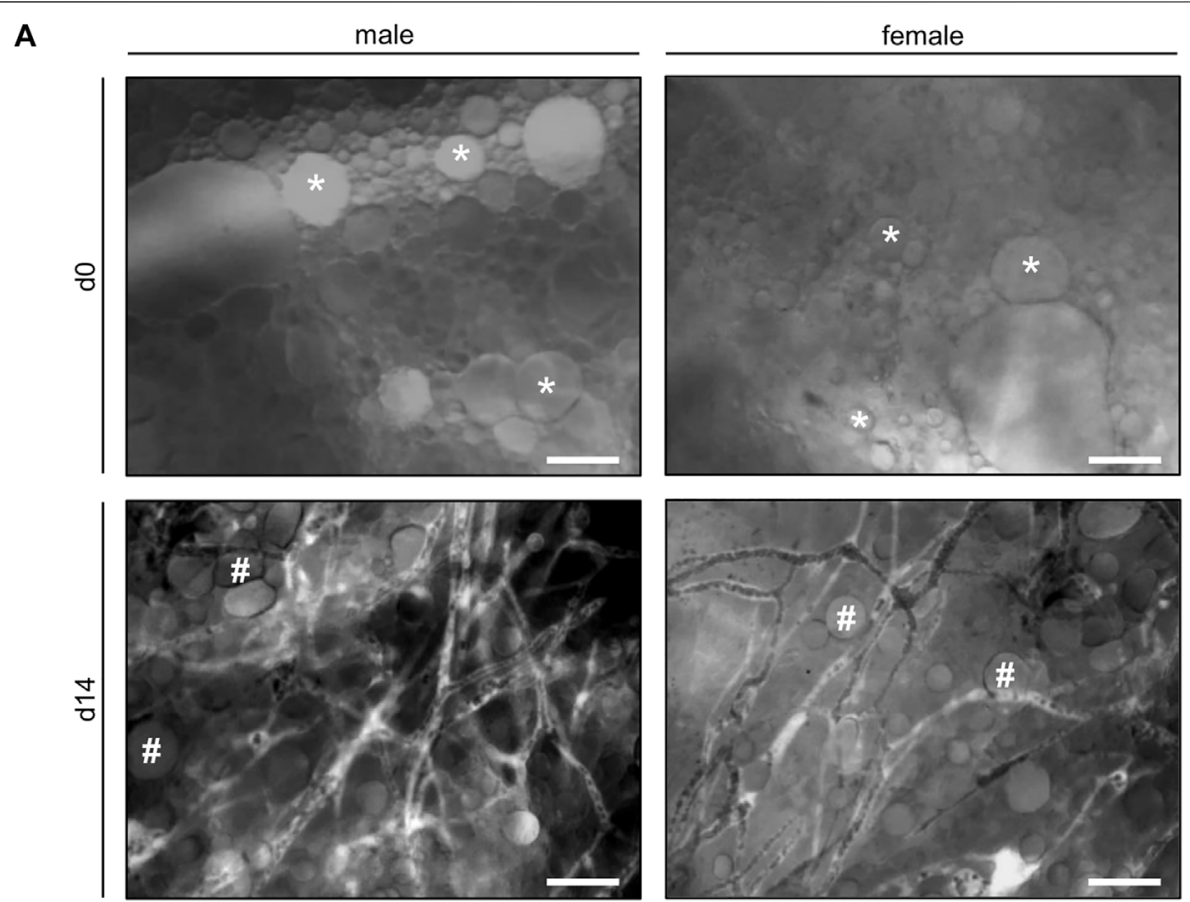

B

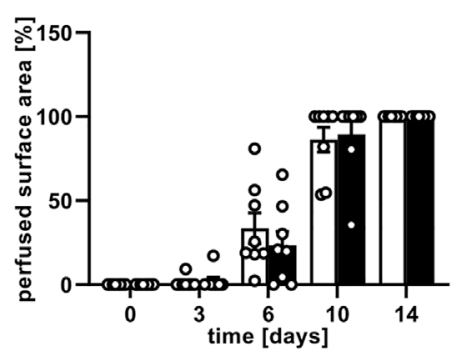

C

D
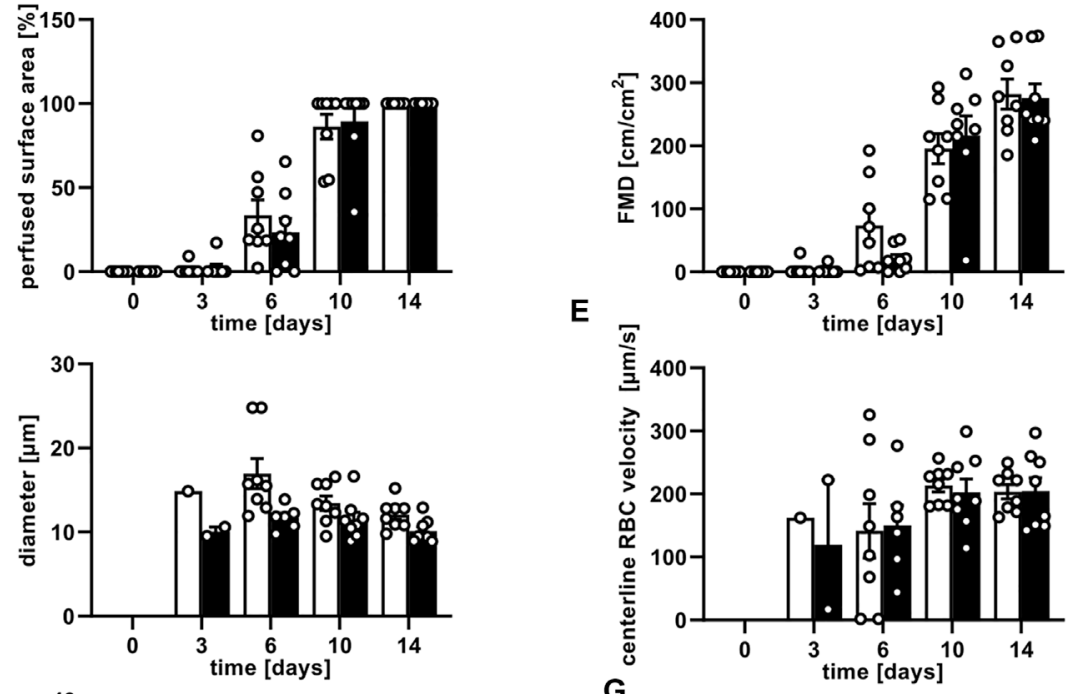

F
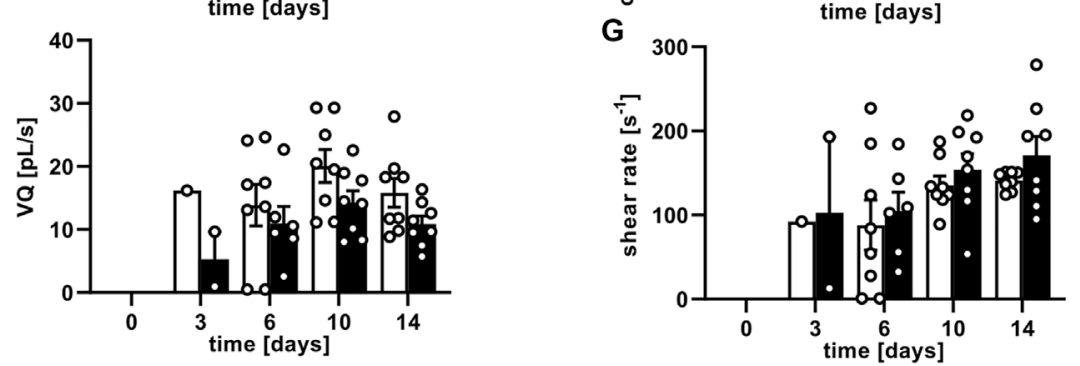

FIGURE 3 | (A) Intravital fluorescence microscopic images of nanofat grafts in a male and a female mouse on day 0 and day 14 after transplantation. On day 0 , the grafts consist of individual cells, fluid or oil vacuoles of varying sizes (asterisks) without a distinct tissue architecture. On day 14, the grafts contain newly formed, microvascular networks interspersed with adipocytes and not yet reabsorbed oil vacuoles (hashtags). Blood perfusion is evidenced by the injection of FITC-labeled dextran for contrast enhancement by staining of blood plasma. Scale bars: $50 \mu \mathrm{m}$. (B-G) Perfused surface area ((B), \%), FMD ((C), cm/cm²), diameter ((D), $\mu \mathrm{m})$, centerline RBC velocity ((E), $\mu \mathrm{m} / \mathrm{s}), \mathrm{VQ}(\mathbf{F}), \mathrm{pL} / \mathrm{s})$ and shear rate $(\mathbf{G}), \mathrm{s}^{-1}$ ) of nanofat grafts in female (white bars; $\left.n=8\right)$ and male (black bars; $\left.n=8\right)$ mice on days $0,3,6$, 10 and 14 after transplantation, as assessed by intravital fluorescence microscopy and computer-assisted image analysis. Means \pm SEM. 
A

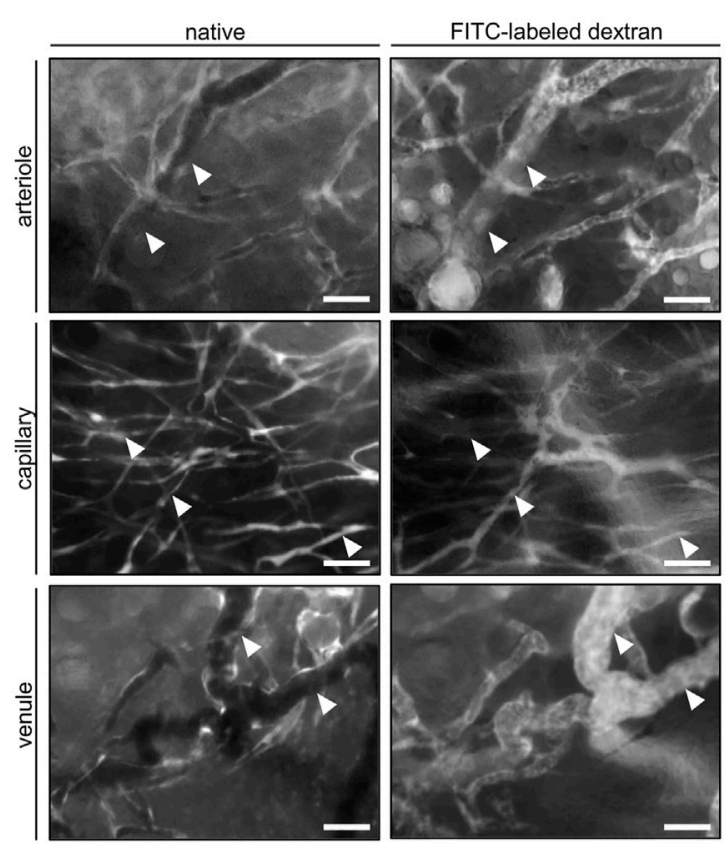

B

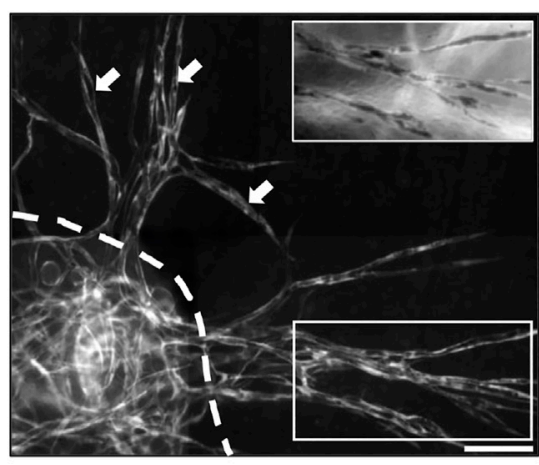

C

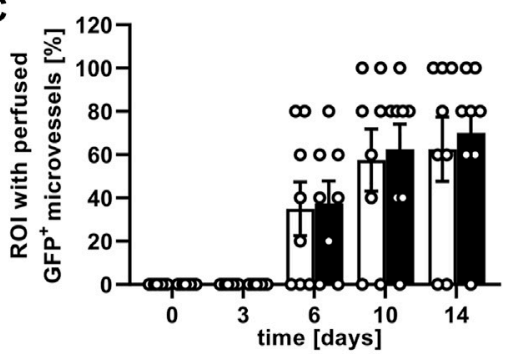

FIGURE 4 | (A) Intravital fluorescence microscopic images of GFP ${ }^{+}$arterioles (arrowheads), capillaries (arrowheads) and venules (arrowheads) before (native) and after the injection of FITC-labled dextran for contrast enhancement by staining of blood plasma on day 14 after transplantation of nanofat into a male mouse. Scale bars: $50 \mu \mathrm{m}$. (B) Intravital fluorescence microscopic images of GFP ${ }^{+}$microvessel sprouts (arrows) growing out of a nanofat graft (border marked by broken line) into the surrounding host tissue on day 14 after transplantation. Insert (border marked by white line) showing the vessel sprouts after the injection of FITC-labeled dextran for contrast enhancement by staining of blood plasma. Scale bar: $50 \mu \mathrm{m}$. (C) Presence of blood-perfused GFP ${ }^{+}$microvessels in the analyzed ROI of nanofat grafts in female (white bars; $n=8$ ) and male (black bars; $n=8$ ) mice, as assessed by intravital fluorescence microscopy. Means \pm SEM.

\section{DISCUSSION}

Over the last decades, the indications for fat grafts in general and nanofat in particular have continuously broadened from a cosmetic to a regenerative use. In fact, the proven effectiveness of nanofat for tissue rejuvenation has led to its rapid clinical success, though its mechanisms of action remain incompletely understood (Menkes et al., 2020; Tonnard et al., 2020). So far, the effects of nanofat have been almost exclusively attributed to its high content of ASCs, which can differentiate into various cell lines (Minteer et al., 2013). In this context, Zhou et al. (2019) demonstrated a significantly upregulated fibroblast differentiation of ASCs, which was induced by co-incubation with adipose extracellular matrix. In a mouse model of skin wounds, these ASCs led to a faster wound healing when compared to extracellular matrix-free ASCs (Zhou et al., 2019). This observation demonstrates that besides ASCs, other nanofat components crucially determine its regenerative properties. In line with this view, we herein demonstrate that nanofat contains functional microvessel segments, which contribute to its capacity to boost tissue vascularization.

Several preclinical animal studies showed an increased vascularization of microfat grafts in mice with elevated serum estrogen levels (Bills et al., 2015; Mok et al., 2018; Wu et al., 2020). In line with these results, we detected a faster onset of nanofat vascularization in the dorsal skinfold chambers of female mice when compared to male animals, as indicated by a slightly larger perfused surface area and FMD of the nanofat grafts on day 6 . However, this did not result in a significantly higher final FMD on day 14 after transplantation. Hence, it may be assumed that nanofat-based vascularization may also not markedly differ between female and male patients under clinical conditions.

The $\mathrm{GFP}^{+} / \mathrm{GFP}^{-}$cross-over design of the present study enabled us to differentiate between $\mathrm{GFP}^{+}$microvessels originating from the nanofat grafts and $\mathrm{GFP}^{-}$microvessels of the host tissue. Of interest, we found that nanofat contains intact microvessel segments, which survive the transplantation procedure and develop into well-organized microvascular networks consisting of arterioles, capillaries and venules. These networks develop interconnections with the microvasculature of the host tissue, resulting in a rapid onset of blood perfusion within the grafts. In addition, they progressively grow out of the grafts and, thus, also improve the vascularization of the surrounding transplantation site. As blood-perfused, nanofatderived $\mathrm{GFP}^{+}$microvessels were detected as early as day 6 after grafting, it is not likely that they differentiated from stem cells within this time. A similar vascularization mode has already been described for adipose tissue-derived microvascular fragments (MVF) (Frueh et al., 2017; Laschke et al., 2021). These MVF can be harvested from fat samples in large amounts and have been proven to be versatile vascularization units for implanted biomaterials (Später et al., 2018; Später et al., 2020), organoids (Nalbach et al., 2021; Strobel et al., 2021) and injured tissues, such as bone and muscle (Pilia et al., 2014; Orth 


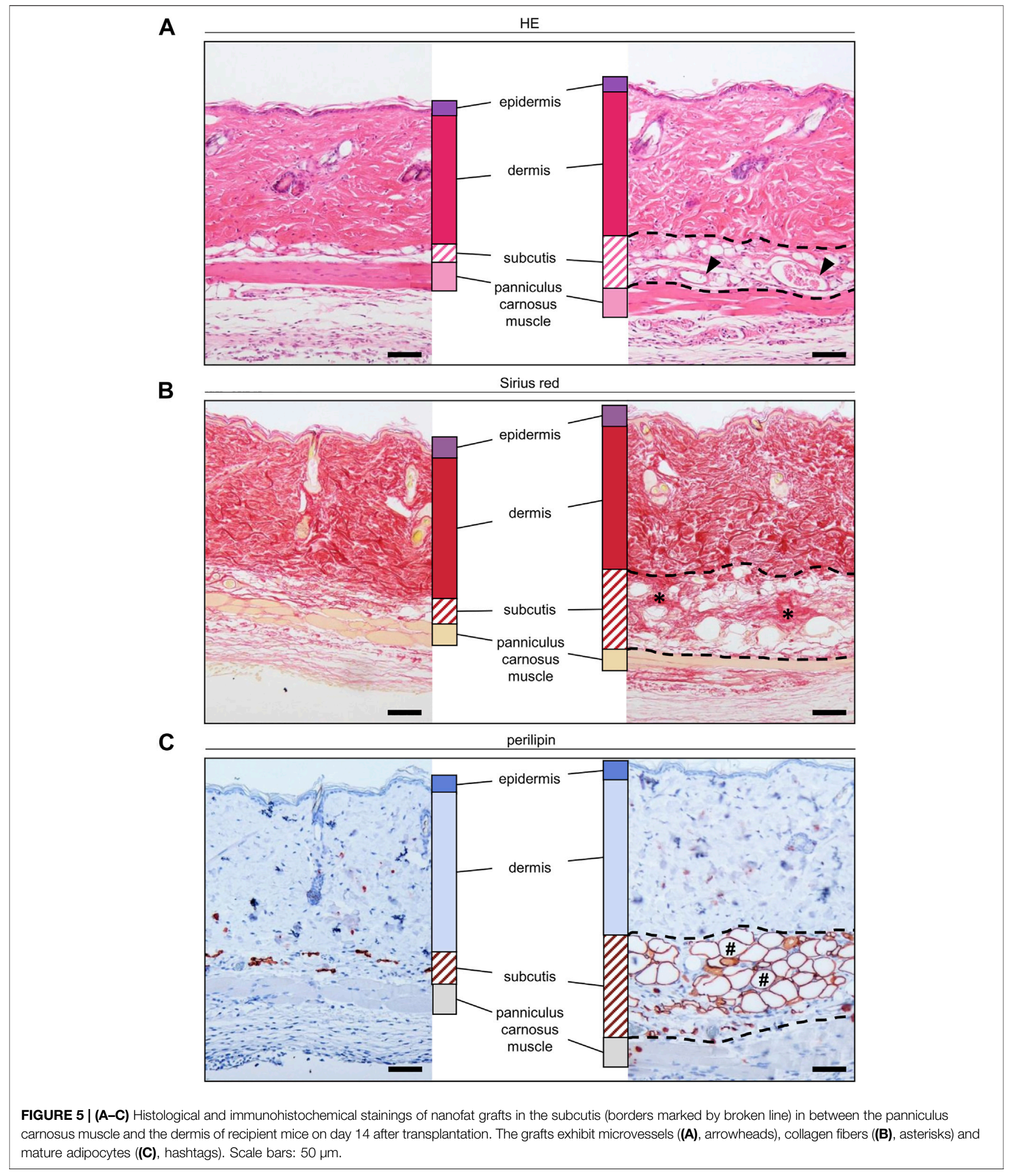

et al., 2018). However, their enzymatic isolation may bear the disadvantage that it requires special expensive equipment and is difficult to standardize due to dissimilar lot-to-lot enzyme activities (Trivisonno et al., 2019). Moreover, enzymatic digestion does currently not meet the requirements for minimal cell manipulation according to regulatory agencies 


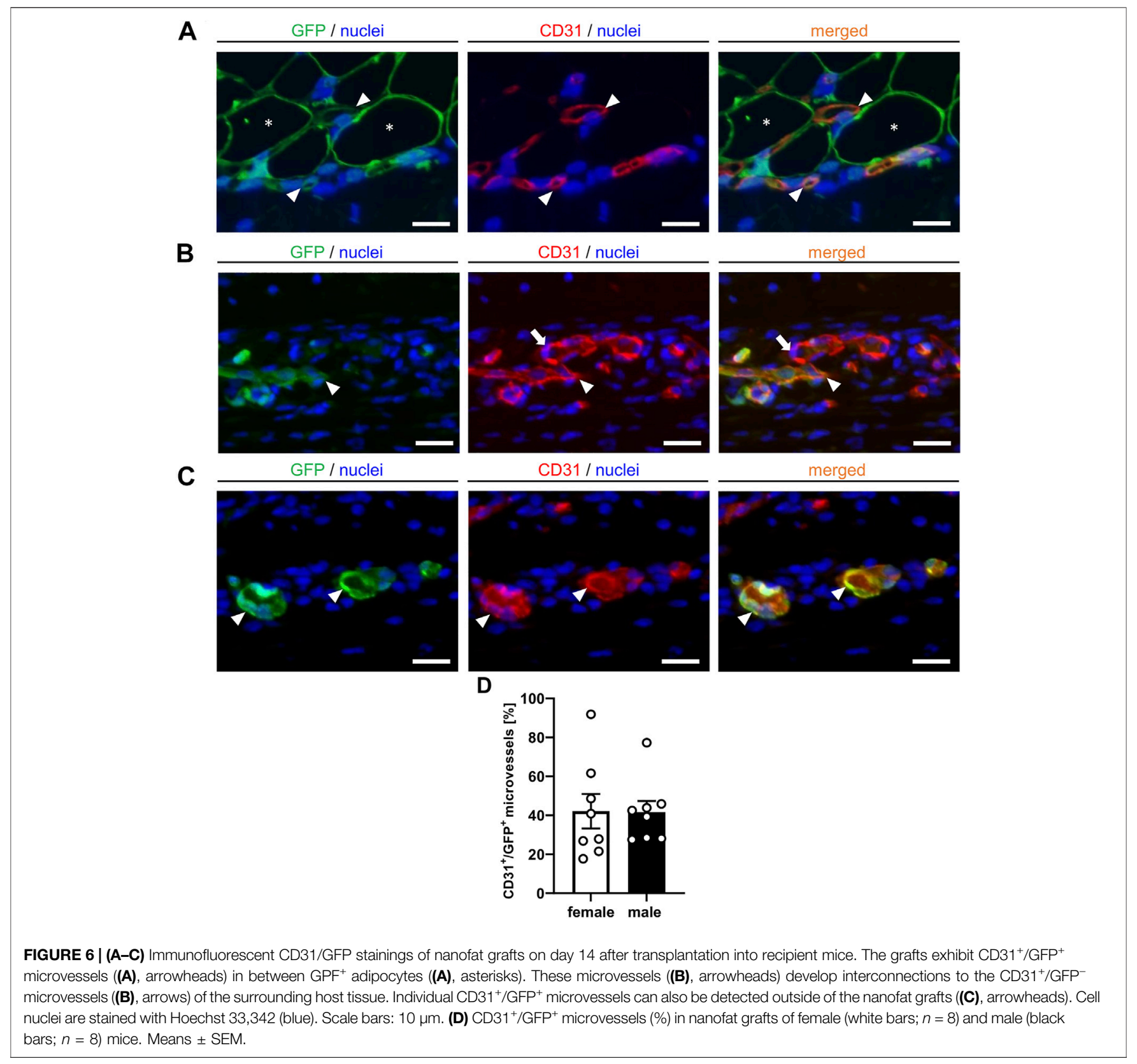

(Raposio and Ciliberti, 2017). In contrast, the inexpensive, quick and easy mechanical generation of nanofat may overcome these hurdles. This may facilitate its widespread use in clinical practice. Although nanofat is a heterogenous mixture of cells, vessel segments and extracellular matrix compounds, it still exhibits a liquid consistency and small particle size (Cohen et al., 2017). Therefore, it may not only be used as a biological, injectable filler, but also for the seeding of biomaterial implants in order to accelerate and improve their tissue incorporation (Grünherz et al., 2019).

At the end of our in vivo experiments, we additionally analyzed the nanofat grafts by means of histology and immunohistochemistry. These analyses revealed that on day 14 after transplantation, the grafts still consisted of microvessels, fibrous tissue and perilipin ${ }^{+}$adipocytes. Although not further examined in the present study, it would be interesting to clarify the fate of these components over longer observation periods in order to identify possible positive or negative effects associated with nanofat grafting. In this context, other long-term studies have already shown that nanofat restitutes dermal thickness after UV light-induced skin damage (Xu et al., 2018) and facilitates tissue remodeling, resulting in physiologically organized collagen and elastin fiber organization (Zheng et al., 2019).

Finally, it should be mentioned that this study also has some limitations. For intravital fluorescence microscopy, we used a conventional epi-illumination microscope with a limited depth of focus. Hence, since in our dorsal skinfold chamber model the 
nanofat was injected into the subcutis underneath the panniculus carnosus muscle, we may have missed microvessels in deeper layers of the grafts and the surrounding host tissue. In future studies this drawback may be overcome by means of confocal microscopy. Moreover, it may be recommended to use a plasma marker in another spectrum than the herein used FITC-labeled dextran. This would bear the advantage that the blood perfusion of $\mathrm{GFP}^{+}$microvessels could be detected in another filter without an overlay of the GFP and FITC signals.

In conclusion, the present study suggests that nanofat is a potential source of functional microvessel segments with a high vascularization capacity. Even though further research is needed to confirm this finding, nanofat grafting may represent a promising approach to boost tissue vascularization. This may open the door for completely new applications of nanofat in the field of tissue engineering and regenerative medicine beyond the scope of aesthetic indications, such as the improvement of wound healing and biomaterial integration.

\section{DATA AVAILABILITY STATEMENT}

The raw data supporting the conclusions of this article will be made available by the authors, without undue reservation.

\section{REFERENCES}

Baker, M., and Wayland, H. (1974). On-line Volume Flow Rate and Velocity Profile Measurement for Blood in Microvessels. Microvasc. Res. 7, 131-143. doi:10.1016/0026-2862(74)90043-0

Banfi, A., Holnthoner, W., Martino, M. M., and Ylä-Herttuala, S. (2018). Editorial: Vascularization for Regenerative Medicine. Front. Bioeng. Biotechnol. 6, 175. doi:10.3389/fbioe.2018.00175

Bills, J. D., Derderian, C., Barker, J., Lowe, A., Lavery, L. A., and Davis, K. E. (2015). The Role of Estrogen in the Modulation of Autologous Fat Graft Outcomes. Plast. Reconstr. Surg. 135, 103e-113e. doi:10.1097/PRS. 0000000000000792

Brüggen, M. C., and Stingl, G. (2020). Subcutaneous white Adipose Tissue: The Deepest Layer of the Cutaneous Immune Barrier. JDDG: J. der Deutschen Dermatologischen Gesellschaft 18, 1225-1227. doi:10.1111/ddg.14335

Chait, A., and den Hartigh, L. J. (2020). Adipose Tissue Distribution, Inflammation and its Metabolic Consequences, Including Diabetes and Cardiovascular Disease. Front. Cardiovasc. Med. 7, 22. doi:10.3389/fcvm.2020.00022

Cohen, S. R., Hewett, S., Ross, L., Delaunay, F., Goodacre, A., Ramos, C., et al. (2017). Regenerative Cells for Facial Surgery: Biofilling and Biocontouring. Aesthet. Surg. J. 37, S16-S32. doi:10.1093/asj/sjx078

De Vriese, A. S., Verbeuren, T. J., Vallez, M.-O., Lameire, N. H., De Buyzere, M., and Vanhoutte, P. M. (2000). Off-line Analysis of Red Blood Cell Velocity in Renal Arterioles. J. Vasc. Res. 37, 26-31. doi:10.1159/000025710

Frueh, F. S., Später, T., Lindenblatt, N., Calcagni, M., Giovanoli, P., Scheuer, C., et al. (2017). Adipose Tissue-Derived Microvascular Fragments Improve Vascularization, Lymphangiogenesis, and Integration of Dermal Skin Substitutes. J. Invest. Dermatol. 137, 217-227. doi:10.1016/j.jid.2016.08.010

Gianni-Barrera, R., Di Maggio, N., Melly, L., Burger, M. G., Mujagic, E., Gürke, L., et al. (2020). Therapeutic Vascularization in Regenerative Medicine. Stem Cells Translational Med. 9, 433-444. doi:10.1002/sctm.19-0319

Grünherz, L., Sanchez-Macedo, N., Frueh, F. S., McLuckie, M., and Lindenblatt, N. (2019). Nanofat Applications: from Clinical Esthetics to Regenerative Research. Curr. Opin. Biomed. Eng. 10, 174-180. doi:10.1016/j.cobme.2019.07.002

Herold, J., and Kalucka, J. (2021). Angiogenesis in Adipose Tissue: The Interplay Between Adipose and Endothelial Cells. Front. Physiol. 11, 624903. doi:10.3389/ fphys.2020.624903

\section{ETHICS STATEMENT}

The animal study was reviewed and approved by Saaarland governmental animal protection committee (permit number: 30/2020).

\section{AUTHOR CONTRIBUTIONS}

AW and ML contributed to the study conception and design. Material preparation, data collection and analysis were performed by AW. The first draft of the manuscript was written by AW and ML. The draft was revised and corrected by YH, DS and MM. All authors read and approved the final manuscript.

\section{ACKNOWLEDGMENTS}

We are grateful for the technical assistance of Caroline Bickelmann and Janine Becker (Institute for Clinical \& Experimental Surgery, Saarland University, Homburg/Saar, Germany).

Illouz, Y.-G. (1986). The Fat Cell "Graft". Plast. Reconstr. Surg. 78, 122-123. doi:10. 1097/00006534-198607000-00028

Laschke, M., Vollmar, B., Vollmar, M. D., and Menger, M. (2011). The Dorsal Skinfold Chamber: Window into the Dynamic Interaction of Biomaterials with Their Surrounding Host Tissue. eCM 22, 147-167. doi:10.22203/ eCM.v022a12

Laschke, M. W., Später, T., and Menger, M. D. (2021). Microvascular Fragments: More Than Just Natural Vascularization Units. Trends Biotechnol. 39, 24-33. doi:10.1016/j.tibtech.2020.06.001

Laschke, M. W., and Vollmar, B. (2018). Vascularization, Regeneration and Tissue Engineering. Eur. Surg. Res. 59, 230-231. doi:10.1159/000492372

Mazzola, R. F., and Mazzola, I. C. (2015). History of Fat Grafting. Clin. Plast. Surg. 42, 147-153. doi:10.1016/j.cps.2014.12.002

Menkes, S., SidAhmed-Mezi, M., Meningaud, J. P., Benadiba, L., Magalon, G., and Hersant, B. (2020). Microfat and Nanofat Grafting in Genital Rejuvenation. Aesthet. Surg. J. 41, 1060-1067. doi:10.1093/asj/sjaal18

Minteer, D., Marra, K. G., and Rubin, J. P. (2012). Adipose-derived Mesenchymal Stem Cells: Biology and Potential Applications. Adv. Biochem. Engineering/ Biotechnology 129, 59-71. doi:10.1007/10_2012_146

Mok, H., Feng, J., Hu, W., Wang, J., Cai, J., and Lu, F. (2018). Decreased Serum Estrogen Improves Fat Graft Retention by Enhancing Early Macrophage Infiltration and Inducing Adipocyte Hypertrophy. Biochem. Biophysical Res. Commun. 501, 266-272. doi:10.1016/j.bbrc.2018.04.232

Nalbach, L., Roma, L. P., Schmitt, B. M., Becker, V., Körbel, C., Wrublewsky, S., et al. (2021). Improvement of Islet Transplantation by the Fusion of Islet Cells with Functional Blood Vessels. EMBO Mol. Med. 13, e12616. doi:10.15252/ emmm.202012616

Orth, M., Altmeyer, M. A. B., Scheuer, C., Braun, B. J., Holstein, J. H., Eglin, D., et al. (2018). Effects of Locally Applied Adipose Tissue-Derived Microvascular Fragments by Thermoresponsive Hydrogel on Bone Healing. Acta Biomater. 77, 201-211. doi:10.1016/j.actbio.2018.07.029

Pilia, M., McDaniel, J. S., McDaniel, J., Guda, T., Chen, X., Rhoads, R., et al. (2014). Transplantation and Perfusion of Microvascular Fragments in a Rodent Model of Volumetric Muscle Loss Injury. eCM 28, 11-24. doi:10. 22203/eCM.v028a02

Raposio, E., and Ciliberti, R. (2017). Clinical Use of Adipose-Derived Stem Cells: European Legislative Issues. Ann. Med. Surg. 24, 61-64. doi:10.1016/j.amsu. 2017.11.002 
Rubanyi, G. M., Johns, A., and Kauser, K. (2002). Effect of Estrogen on Endothelial Function and Angiogenesis. Vascul Pharmacol. 38, 89-98. doi:10.1016/S03063623(02)00131-3

Später, T., Frueh, F. S., Nickels, R. M., Menger, M. D., and Laschke, M. W. (2018). Prevascularization of Collagen-Glycosaminoglycan Scaffolds: Stromal Vascular Fraction versus Adipose Tissue-Derived Microvascular Fragments. J. Biol. Eng. 12, 24. doi:10.1186/s13036-018-0118-3

Später, T., Tobias, A. L., Menger, M. M., Nickels, R. M., Menger, M. D., and Laschke, M. W. (2020). Biological Coating with Platelet-Rich Plasma and Adipose Tissue-Derived Microvascular Fragments Improves the Vascularization, Biocompatibility and Tissue Incorporation of Porous Polyethylene. Acta Biomater. 108, 194-206. doi:10.1016/j.actbio.2020.03.018

Strobel, H. A., Gerton, T., and Hoying, J. B. (2021). Vascularized Adipocyte Organoid Model Using Isolated Human Microvessel Fragments. Biofabrication 13, 035022. doi:10.1088/1758-5090/abe187

Tonnard, P., Verpaele, A., and Carvas, M. (2020). Fat Grafting for Facial Rejuvenation with Nanofat Grafts. Clin. Plast. Surg. 47, 53-62. doi:10.1016/j. cps.2019.08.006

Tonnard, P., Verpaele, A., Peeters, G., Hamdi, M., Cornelissen, M., and Declercq, H. (2013). Nanofat Grafting. Plast. Reconstr. Surg. 132, 1017-1026. doi:10.1097/ PRS.0b013e31829fe1b0

Trivisonno, A., Alexander, R. W., Baldari, S., Cohen, S. R., Di Rocco, G., Gentile, P., et al. (2019). Intraoperative Strategies for Minimal Manipulation of Autologous Adipose Tissue for Cell- and Tissue-Based Therapies: Concise Review. Stem Cells Translational Med. 8, 1265-1271. doi:10.1002/sctm.19-0166

Wu, S., Zhu, Y., Zhang, J., Hu, X., and Yi, Y. (2020). Effect of Circulating Estrogen Level on the Outcome of Free Fat Grafting in Nude Mice. Zhongguo Xiu Fu Chong Jian Wai Ke Za Zhi 34, 220-225. doi:10.7507/1002-1892.201903011

Xu, P., Yu, Q., Huang, H., Zhang, W. J., and Li, W. (2018). Nanofat Increases Dermis Thickness and Neovascularization in Photoaged Nude Mouse Skin. Aesth Plast. Surg. 42, 343-351. doi:10.1007/s00266-018-1091-4
Zheng, H., Qiu, L., Su, Y., and Yi, C. (2019). Conventional Nanofat and SVF/ ADSC-Concentrated Nanofat: A Comparative Study on Improving Photoaging of Nude Mice Skin. Aesthet. Surg. J. 39, 1241-1250. doi:10.1093/asj/sjz066

Zhou, Z. Q., Chen, Y., Chai, M., Tao, R., Lei, Y. H., Jia, Y. Q., et al. (2019). Adipose Extracellular Matrix Promotes Skin Wound Healing by Inducing the Differentiation of Adipose-derived S-tem C-ells into F-ibroblasts. Int. J. Mol. Med. 43 (2), 890-900. doi:10.3892/ijmm.2018.4006

Zuk, P. A., Zhu, M., Ashjian, P., De Ugarte, D. A., Huang, J. I., Mizuno, H., et al (2002). Human Adipose Tissue Is a Source of Multipotent Stem Cells. MBoC 13, 4279-4295. doi:10.1091/mbc.E02-02-0105

Zuk, P. A., Zhu, M., Mizuno, H., Huang, J., Futrell, J. W., Katz, A. J., et al. (2001). Multilineage Cells from Human Adipose Tissue: Implications for Cell-Based Therapies. Tissue Eng. 7, 211-228. doi:10.1089/107632701300062859

Conflict of Interest: The authors declare that the research was conducted in the absence of any commercial or financial relationships that could be construed as a potential conflict of interest.

Publisher's Note: All claims expressed in this article are solely those of the authors and do not necessarily represent those of their affiliated organizations, or those of the publisher, the editors and the reviewers. Any product that may be evaluated in this article, or claim that may be made by its manufacturer, is not guaranteed or endorsed by the publisher.

Copyright (c) 2022 Weinzierl, Harder, Schmauss, Menger and Laschke. This is an open-access article distributed under the terms of the Creative Commons Attribution License (CC BY). The use, distribution or reproduction in other forums is permitted, provided the original author(s) and the copyright owner(s) are credited and that the original publication in this journal is cited, in accordance with accepted academic practice. No use, distribution or reproduction is permitted which does not comply with these terms. 